\title{
The influence of objective measurement tools on communication and clinical decision making in neurological rehabilitation
}

\author{
Sarah F. Tyson PhD MCSP, Joanne Greenhalgh PhD, ${ }^{2}$ Andrew F. Long $\mathrm{PhD}^{3}$ and Robert Flynn $\mathrm{PhD}^{4}$ \\ ${ }^{1}$ Professor of Rehabilitation, School of Health, Sport and Rehabilitation Sciences, University of Salford, Salford, UK \\ ${ }^{2}$ Senior Research Fellow, School of Healthcare, University of Leeds, Leeds, UK \\ ${ }^{3}$ Professor of Health Care Practice, School of Healthcare, University of Leeds, Leeds, UK \\ ${ }^{4}$ Professor of Sociology, Institute Social, Cultural and Policy Research, University of Salford, Salford, UK
}

\begin{abstract}
Keywords
clinical decision making, communication, neurological rehabilitation, outcome measures
\end{abstract}

\author{
Correspondence \\ Professor Sarah Tyson \\ School of Health \\ Sport and Rehabilitation Sciences \\ Frederick Road Campus \\ University of Salford \\ Salford M6 6PU \\ UK \\ E-mail: s.tyson@salford.ac.uk
}

Accepted for Publication: 28 June 2010

doi:10.1111/j.1365-2753.2010.01555.x

\begin{abstract}
Rationale Routine collection of outcome measures is advocated to improve quality of care. However, there has been scant investigation of how measurement tools are used in clinical practice and what impact they may have. This paper compares two neurorehabilitation teams, one which routinely used standardized measurement tools and the other which did not. We explore differences in communication and clinical decision making within multidisciplinary team (MDT) meetings to illuminate the influence measurement tools could have on clinical practice.

Method Non-participant observation of MDT meetings in two neurological rehabilitation units in England. Semi-structured interviews were also carried out with at least one member of each profession in each team. Grounded theory techniques were used to analyse the data. Results Differences in team members' communication within MDT meetings underscored differences in the process of clinical decision making within the teams. Using measurement tools provided a shared understanding to facilitate communication by focusing discussion on the patient's abilities rather than individual professionals' contributions. This led to differences in the way team members identified the nature and cause of patients' problems, monitored their progress and planned for discharge. They provided a 'neutral ground' to reach a shared perspective between professionals, thereby avoiding conflict. Externally, use of the tools enabled objective discussion with patients and their families about their progress and was a vehicle to facilitate giving bad news.

Conclusion Using standardized measurement tools can promote a patient-focused approach to care, thus facilitating treatment planning and clinical decision making.
\end{abstract}

\section{Introduction}

The routine collection of outcome measures has been advocated as an essential component of evidence-based practice and a means of improving patient outcomes [1-5] and, more recently, has been highlighted as a way to monitor and improve quality of care [6-9]. Despite this political drive and a huge literature on the psychometric development of different measurement tools, there has been very little investigation of how measurement tools are used in clinical practice and what influence they have on processes of care.

Most research in this area has focused on the collection and feedback patient-reported outcome measures (PROMs) to clinicians at the patient clinician interface [10]. It suggests that while PROMs increase the extent to which clinicians identify patient's problems and discuss health-related quality of life issues with patients, they have little influence on how such problems are managed or ultimately, patient's health. However, this literature has treated clinical decision making as a 'black box' by focusing on whether the collection of outcomes information makes a difference to the treatment and health outcomes of patients and ignoring how clinicians make use of the information from outcome measures within decision making [11].

Furthermore, many patients, such as those with long-term or complex conditions in rehabilitation settings, are cared for by multidisciplinary teams (MDTs) in which communication and decision making is a key component of care. A few nonrandomized studies have examined the impact of standardized outcome measures on MDT working in rehabilitation settings, 
finding that they did not improve health outcomes but did have a modest benefit on patient satisfaction [12,13]. Qualitative studies have found that clinicians perceive that the use of a measurement tool improved client participation in rehabilitation and helped to focus the MDT on client needs [14].

Earlier papers from our own work have examined the use of measurement tools by an MDT which routinely use standardized measurement tools in their daily clinical practice [15-17]. We drew attention to how this MDT used the tools internally, to aid communication within the MDT and plan treatment and externally to support communicating with patients, families and other service providers, to justify the service and to enable audit and service development. This paper explores and compares the approach taken by this team, which used standardized measurement tools, with another team which did not routinely use them. We explore differences in communication and clinical decision making within MDT meetings between the two teams to illuminate the influence the use of measurement tools could have on clinical practice.

\section{Method}

\section{Setting}

Neuro-rehabilitation was chosen as a setting to explore the influence of outcome measures on team clinical decision making for two reasons. First, neuro-rehabilitation teams include clinicians from a range of professional backgrounds, including medicine, nursing, physiotherapy, speech therapy, psychology and social work. This enables observation of whether and how outcome measures facilitate communication between clinicians from different backgrounds. Second, the collection of outcome measures in neuro-rehabilitation is relatively common. Around $85 \%$ of neurorehabilitation teams in the UK collect at least one standardized outcome measure as part of routine patient care [18].

Two adult neurological rehabilitation units of similar size were purposefully selected. One was part of a large teaching National Health Service (NHS) trust and routinely collected standardized measurement tools (Unit 1). The other located in a satellite unit near a large district general hospital (Unit 2). Both units were NHS Foundation Trusts and the service was led by a consultant in rehabilitation medicine and had a dedicated MDT. Involvement in the study was agreed by the MDT who all gave written informed consent. University and NHS ethical approval and local R\&D approval were obtained.

\section{Data collection}

Data were collected by JG using non-participant observation of MDT meetings and semi-structured interviews with members of the MDT. In both units, the function of the MDT meetings was to identify patient's problems, co-ordinate care, review the patients' progress and plan discharge. It was in these meetings that measurement tools were discussed in Unit 1. The format of the meetings in each unit is shown in Table 1.

The MDT meetings were observed over 8 weeks in Unit 1 $(n=14)$ and over 7 weeks in Unit $2(n=7)$. A total of 60 patients were considered (39 in Unit 1 and 21 in Unit 2) and eight complete patient journeys (from admission to discharge) were covered (five in Unit 1 and three in Unit 2). Field notes were made during each meeting and analytical memos developed as the fieldwork progressed. Towards the end of the observation period, at least one clinician from each profession in the MDT was interviewed (11 in Unit 1 and 8 in Unit 2) to explore team members' perceptions of the role and influence of measurement tools in clinical decision making, their experience of using measurement tools, the challenges or limitations and the factors that influenced these. Both the MDT meetings and the interviews were audiotaped and subsequently transcribed.

\section{Analysis}

A grounded theory approach was used to analyse the data and was iterative and ongoing throughout the study [19,20]. Our analysis had a two-stage approach: a within-site analysis followed by a comparative analysis across sites. Our within site analysis involved repeated readings of the field notes, memos and transcripts of MDT meetings. We initially developed broad open codes to describe key aspects of clinical decision making including how patients' problems were identified and discussed, how progress was monitored and discharge planned. To test out our initial hypotheses in more detail, we constructed 'patient journeys'. These consisted of all the data (from transcripts and field notes) about a particular patient during successive MDT meetings (from admission to discharge) plus any relevant data from the interviews conducted with team members.

To facilitate in-depth comparison between the units, we selected two 'pairs' of patient journeys of patients with similar characteristics from each site. One pair [patients 14 (Unit 1) and 41 (Unit 2)] had severe brain injuries, were completely dependent and thought by the staff to have little potential for recovery. In both cases, this latter view contrasted with that of their spouses, who felt they could make progress with more rehabilitation. The other pair [patients 21 (Unit 1) and 51 (Unit 2)] had mild-to-moderate physical impairments and activity limitations but severe cognitive impairments which limited independence in the activities of daily living and caused behavioural and communication difficulties.

We then developed codes to conceptualize what was different about the ways in which the key aspects of clinical decision making explored in our within site analysis were discussed in Unit 1 compared with Unit 2 and more specifically, how and why the use of measurement tools might explain these differences. Emerging findings were discussed and refined through regular meetings between the authors. Data were managed using the qualitative software package, QSR N-Vivo v2.0.

\section{Results}

\section{Overview}

We found that differences in team members' communication within MDT meetings underscored differences in the process of clinical decision making within the two teams. These differences were, in part, a function of variations in meeting structure between the sites; however, use of the measurement tools provided a shared framework to facilitate communication within team members. This, in turn, led to differences in the way team members identified patients' problems, monitored their progress and planned for discharge. Below, we discuss these findings in more detail. 
Table 1 Comparison of the structure and format of the multidisciplinary team meetings

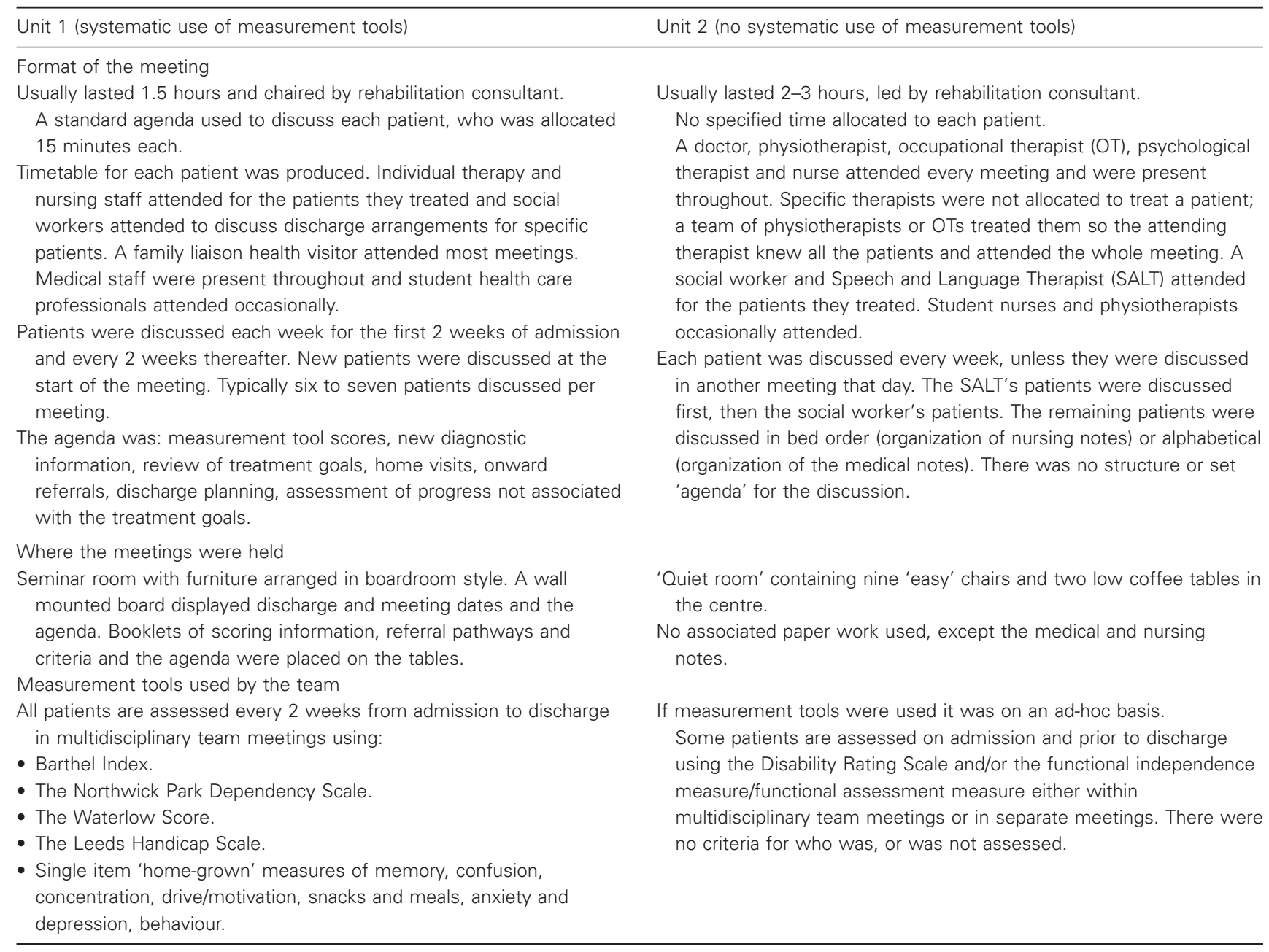

\section{Communication: the structure of the meeting}

In Unit 1, the MDT meeting was patient-focused. As detailed in Table 1, team members followed a structured agenda that focused on the patient's care pathway. The scores from the measurement tools provided a shorthand way of summarizing the patient's abilities and often no further explanation or discussion about them was needed as the MDT held a shared tacit knowledge of what the score meant [17]. When there was further discussion about the scores, it focused on the patient's problems, typically what the patient could and could not do within the context of the items from the measurement tools. To progress the discussion, the consultant (who chaired the meetings) would usually ask for the scores for the next set of the patients' problems.

In contrast, the meeting in Unit 2 focused on the contribution of each profession. Each team member would report their progress and impression of the patient with little 'cross-talk' between the professions. The consultant chairing the meeting would often ask about 'the physio input' or for the 'nursing aspects' to move the conversation on. The reportage focused on descriptions of the patient's behaviour or the therapists' activities in subjective terms and was often lengthy and repetitive. Problem identification was dispersed among more general discussion and rarely pulled together.

\section{Communication: avoiding conflict}

In Unit 2, as the different professions reported their input, different opinions or experiences were sometimes reported or different observations made. These were rarely acknowledged and few attempts were made to resolve the differences. Rather, a member of the team (usually the consultant chairing the meeting) tended to deal with this by changing the subject to consider another aspect of the patient's problems. This is illustrated in the following extract in which the team's experience of Patient 41's problems are discussed (Extract 1).

One way of interpreting this is as a way to avoid disagreements and conflict within the team, as to question a colleague's report would challenge their professional opinion. In contrast, Unit 1 used scores from the measurement tools to provide a "neutral ground' in which differences of opinion could be debated but attributed to the interpretation of the score, focusing on the 


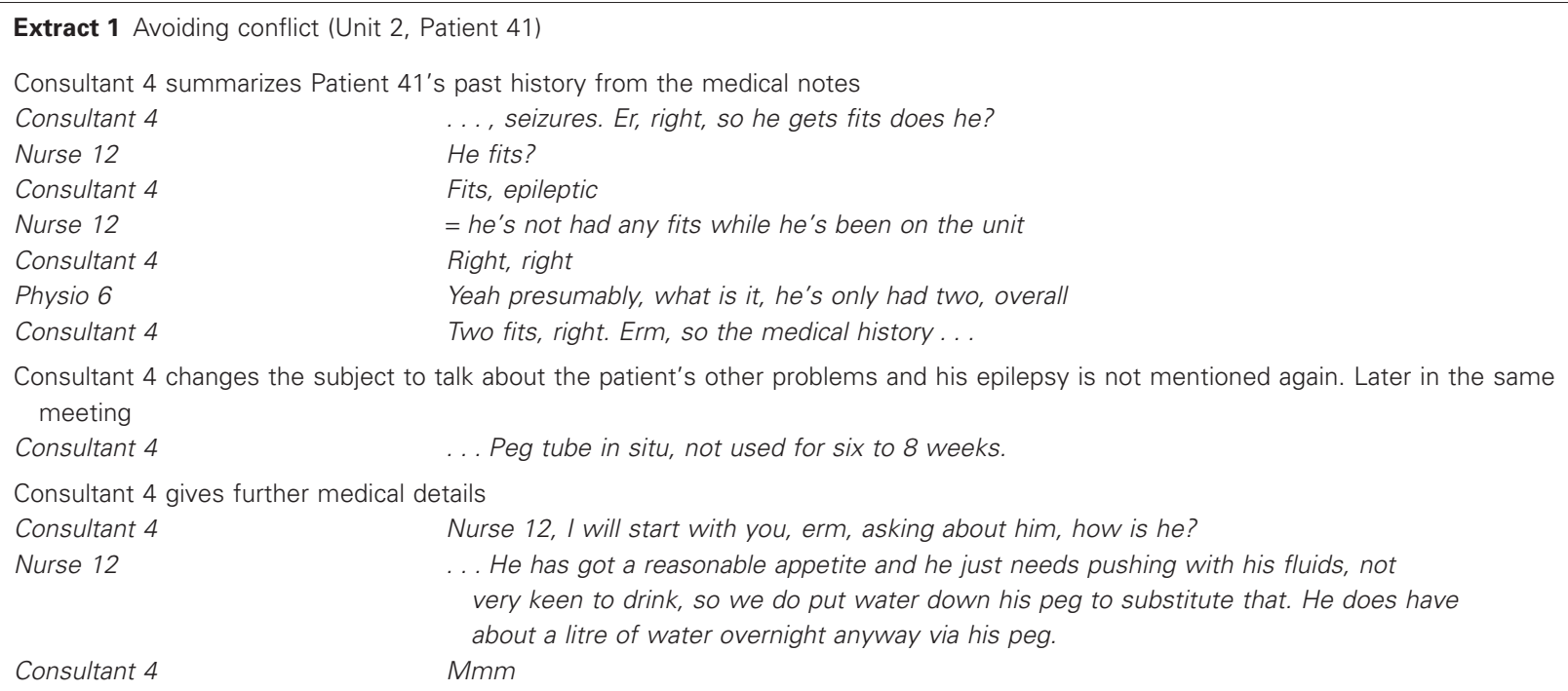

patient's problem rather than professional judgement or contribution to negotiate a shared conclusion, as explained one of the nurses in Unit 1.

We don't normally disagree that much [about the scoring]. We can usually work it out between us and come up with the right answer (Nurse 1, Unit 1).

\section{Communication: dealing with uncertainty}

One of the challenges of neurological rehabilitation is that patients' levels of performance often fluctuate making it difficult to define the precise level of ability and to monitor progress. In Unit 2, the variability of patients' performance was a frequent aspect of the reporting. For example, in one meeting when the team discussed Patient 41's ability to transfer from one chair to another (a component of many activities of daily living) seven different ways in which he performed the activity were reported (off the bed with two assisting; pumping his legs; other transfers with two and quad sticks, or two people assisting; use of rotostand; use of chair; use of hoist). Although the same issue was being raised in different ways, there was little attempt to pull these views together to gain a shared picture or to summarize the patient's abilities.

In contrast, in Unit 1, the patient's ability to transfer was scored using the transfer section of the Barthel Index. If this scale had been applied to Patient 41 (of Unit 2), his score would have been 'zero', indicating that he needed two people to assist him to transfer. In Unit 1, this would have been be stated (and negotiated if necessary) once at the start of the meeting and would not need to be referred to again, resulting in a succinct summary, with a common meaning known to the whole MDT. To aid this process, the MDT in Unit 1 had developed 'rules of thumb' to decide how the scores should be decided if the patient's performance varied. They always gave the score based on what the patient did in everyday life on the ward and if in doubt gave the lower score. This process was used when Patient 14's progress was discussed. The occupational therapist gave a score for the dressing item on the Barthel Index, but qualified it by saying 'he's so variable' and the registrar cautioned 'if you've qualms, it [the score] must be low'. This process was also highlighted by the nurse and the physiotherapist in their interviews.

Some patients do react differently. They could do a perfect transfer in physio, but then on the ward they might not be very good... [to do the scores] we think over the whole week, what they've been like. (Nurse 1, Unit 1)

it tends to be what they do on the ward, it might not be what you want them to do or what they're capable of doing, but what they actually do ... (Physiotherapist 3, Unit 1)

\section{Communication with the patient and family}

Previously, we described how the team in Unit 1 used the measurement tools to communicate with external parties such as the patient and their family [15]. They described how the tools were used to explain the patient's problems to help them and/or their family understand complex problems such as cognitive impairments and their effect on everyday life, and to give feedback about their progress. The tools were also used as 'objective' evidence to support the team's clinical impression of a lack of progress within discussions with family members.

The team in Unit 2 found it difficult to communicate bad news about patients' progress to the patient or their family, particularly when this contrasted with the family's view. In his interview, Consultant 4 described how this difference of opinion impacted negatively on the relationship between the team and the patient and family as it drew the team 'into this attitude of defending or justifying everything you do. I don't think that this is useful or helpful' so that 'We [the team and the family] aren't partners any more, we are sitting on the opposite side of the table'. However, he did not feel that using objective measurement tools would help the situation as the family would not be able to interpret the scores. 


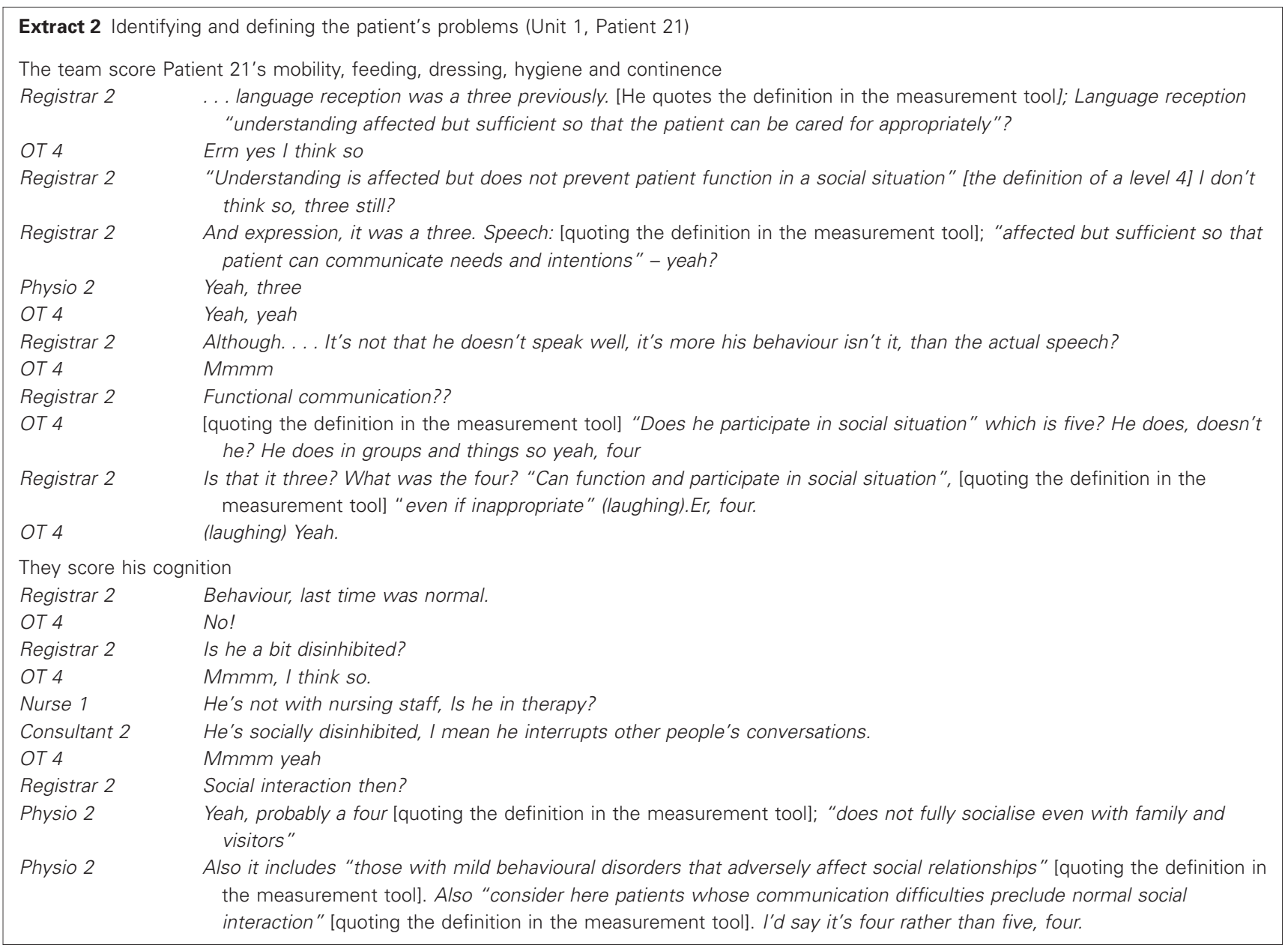

If you try and be very, you know, academic and, you know, the scores are 28 and after 7 months they are 26 . What does this mean to them [the family]? Nothing. For me and the therapist a change of 2 or 3 might mean something. Um, for the family it might not mean anything at all.

$\mathrm{He}$ also felt that it would be difficult to appear to value the impersonal information from the measurement tools over the feelings of the family.

often you come across a family who think the patient is, you know, talking and moving and communicating, where the objective measures might tell you otherwise. It's difficult to, you know, go along with the outcome measure and not to try and please the family if you can.

\section{Clinical decision making}

These differences in communication underscored differences in key aspects of clinical decision making between the teams. In a previous paper [15], we identified three ways in which the use of measurement tools in Unit 1 assisted clinical decision making in terms of:

- Identifying the presence and severity of the patient's impairments and activity limitations.
- Informing treatment planning and monitoring the patient's progress.

- Predicting the patient's recovery and discharge destination.

We now illustrate how the presence or absence of measurement tools influenced how the two units managed these decisions within MDT meetings.

\section{Clinical decision making: identifying and defining the patient's problems}

A key task faced by MDTs at the start of rehabilitation is to arrive at an understanding about the nature, severity and cause of patients' problems and to agree on a course of action to address them. A defining feature of patients receiving specialist neurological rehabilitation is that they have multiple, complex, often interacting problems which rarely fall solely into the remit of a single profession. Consequently, reaching a consensus among the team about their cause and appropriate treatment is not only a priority but also a challenge. To illustrate how this was managed in the two units, we present a comparison of how they discussed the behavioural problems exhibited by Patient 21 (Unit 1) (Extract 2) and Patient 51 (Unit 2) (Extract 3).

In Unit 1, team members used the definitions from the measurement tools to discuss the nature and severity of the Patient 21's 


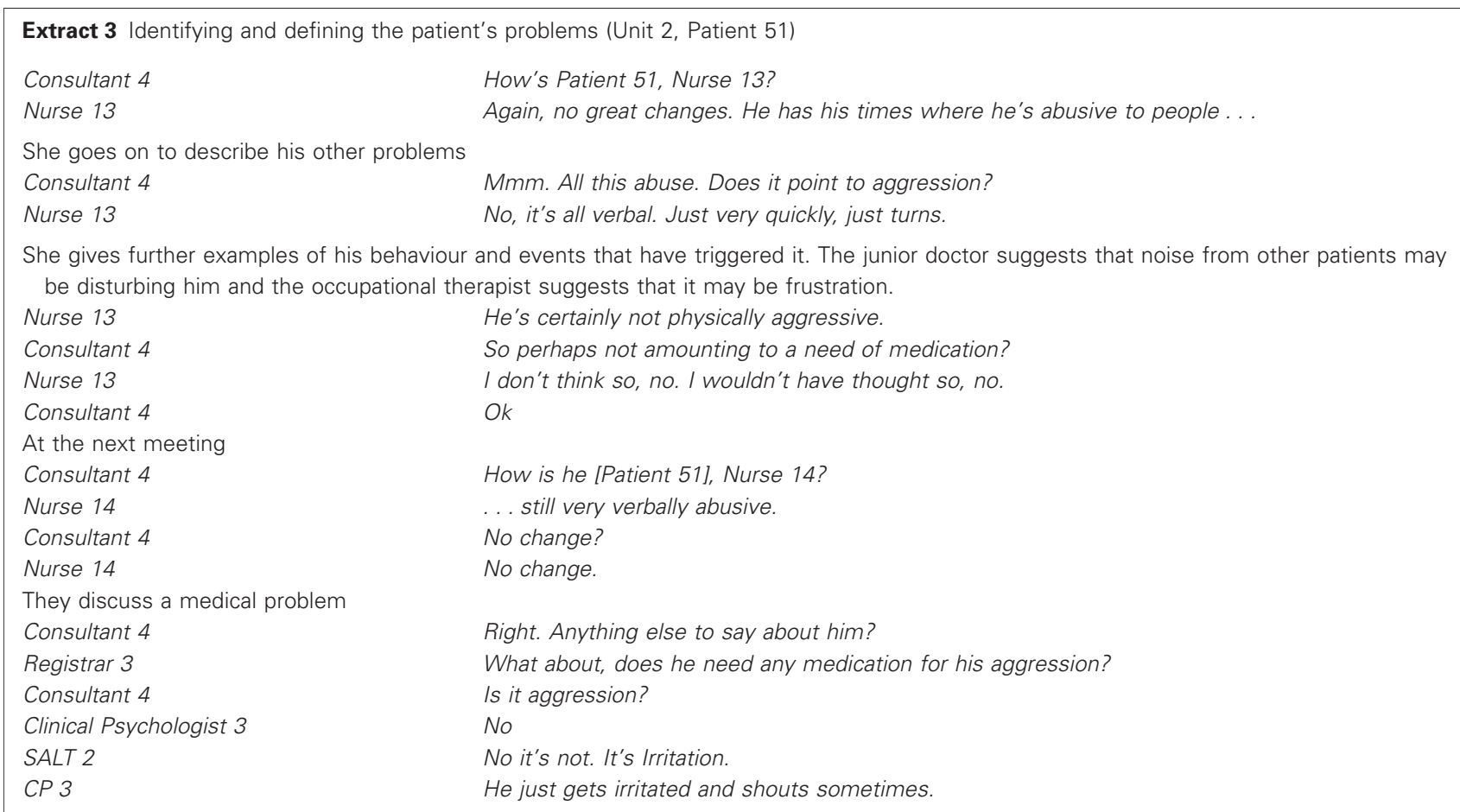

social and behavioural problems, identify and agree on their cause and jointly negotiate an agreed score. As a consultant summarized:

I don't think it's the total score that makes a difference so much as making sure that we cover specific key issues that can influence outcome. It highlights for us where we need to focus more work or find some external support because something cannot be achieved. It's an aide memoire for the team process. (Consultant 2, Unit 1)

By using these definitions, the measurement tools provided a structure and common ground for discussion and once agreement had been achieved, by virtue of their shared tacit understanding of the meaning and implications of the scores [17], sharing information about the patient's abilities simply involved reciting the score allocated to the patient.

In Unit 2, information exchange focused on descriptions of the patient without synthesis to gain a shared view. As Extract 3 shows, different clinicians held different beliefs about the nature and cause of Patient 51's aggression. In particular, the medical staff labelled his behaviour as 'aggressive' and felt that it required medication while the clinical psychologist and the speech therapist viewed this behaviour as 'irritation' and a natural reaction to his situation that did not require any treatment. This difference of opinion persisted over several meetings and no consensus about the cause of the patient's problems or appropriate action was reached.

\section{Clinical decision making: monitoring progress}

As we have highlighted previously [16], fluctuations in the patient's level of performance make it difficult to define their precise level of ability and to monitor progress. The external, standardized definitions of patients' problems offered by the measurement tools enabled Unit 1 to monitor the patients' progress at each meeting by comparing the current scores with those from the previous meeting(s). Extract 4 shows how they discussed Patient 14 's progress in dressing over time. The scores provided a basis on which team members could negotiate the meaning of any changes, whether it was a true change in the patient's abilities or whether it indicated an adjustment in the understanding of the patient's problems [15-17].

The value of the measurement tools when monitoring progress was identified by the staff in Unit 1 .

The big thing about the Barthel is that it informs us on a fortnightly basis whether somebody's still making progress ... if you don't check these things then people will stay in hospital for months and years (Occupational Therapist 4, Unit 1)

They are useful when you see a change from the 2 weeks before. (Nurse 1, Unit 1).

In Unit 2, there were no standardized benchmarks against which progress (or the lack of it) could be assessed. Extract 5 shows how the team discussed Patient 41 's progress regarding mobility over a series of meetings. As it shows, the team often used non-specific descriptions such as 'a bit brighter' or 'not much change' or 'doing a bit more' or provided a description of their activities with the patient. There were few comparisons with assessments made at previous meetings. The basis on which judgements about progress were made sometimes varied between team members or from one meeting to the next. Establishing a shared picture of the change in the patient was also hampered by differing reports of what the patient was able to do in therapy sessions compared with what they were able to do on ward. 


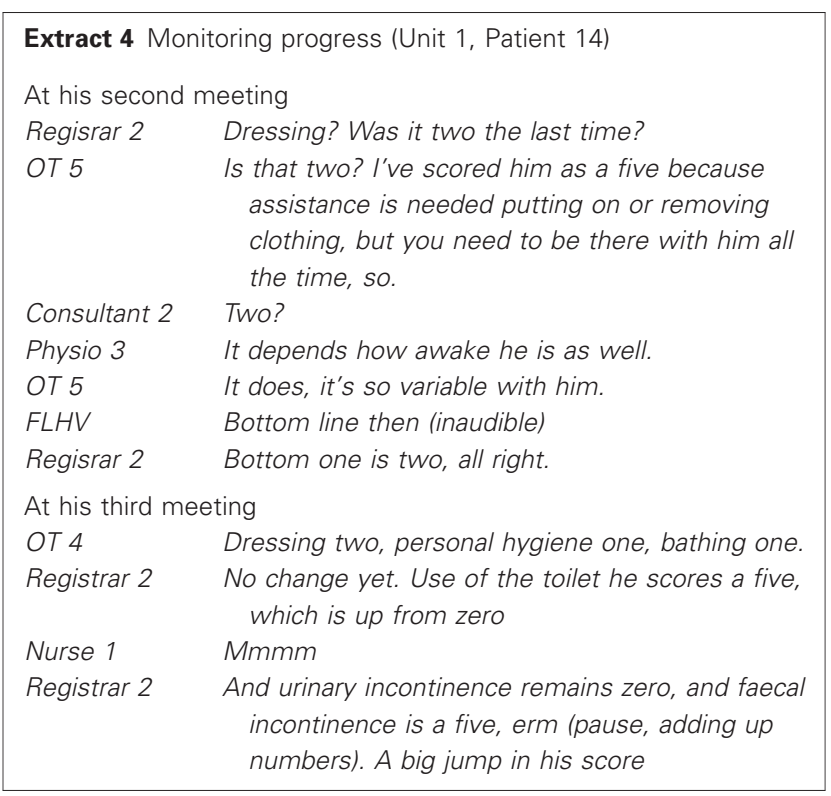

\section{Clinical decision making: predicting recovery and discharge destination}

Discharge planning in rehabilitation is a complex process, often requiring co-ordination of multiple agencies and stakeholders, which was evident in both units we observed. However, in Unit 1, the measurement tools were used soon after admission to inform judgements about the patient's likely response to rehabilitation, the amount of support they might need on discharge and their likely discharge destination. Consultant 1 in Unit 1 explained this process.

I think they [the measurement tools] are a trigger for us at certain stages in the rehabilitation process ... then we can then think about the next stage and er towards planning a discharge.

The following extract shows how the MDT used the scores from the measurement tools to predict Patient 14's likely discharge destination in the first meeting after his admission (Extract 6).

In the absence of evidence provided by measurement tools, the team in Unit 2 relied on what they 'sensed' the patient was, or was not, able to do. They did not feel able to predict how a patient might respond to rehabilitation or a possible discharge destination as the patient's course of recovery was felt to be highly individualized and unpredictable. The clinical psychologist in Unit 2 explained this process. He explained how the team waited until they 'noticed' the patient was no longer changing before making any decisions or plans about discharge.

It's about how much change they [the physios and occupational therapists] notice. They might notice change in things they can do. How long he can engage in an activity for? How far he can extend his arms? How long he's staying awake even? They will work on what they see is improving, but there'll come a time when they think he's not changed for the last however many weeks; that he's plateaue'd. But this idea of plateau, it's a person changing. Are they improving? A person can level off for a little while and then pick up again and so we tend to give it a length of time. That varies according to pressure on beds, professional judgement and also how this person's been medically. That's sort of how discharge planning starts and then it's taken to the MDT meeting. (Clinical Psychologist 3, Unit 2)

Thus, the key issue for the team when deciding whether the patient was ready for discharge was whether they were felt to be benefiting from being in the unit, rather than achieving functional goals. Discharge planning did not begin until the middle or end of rehabilitation after a long trial of treatment. This was explained by the physiotherapist in Unit 2 during his interviews.

Interviewer: When do you know when a person is ready to go?

Physio 6: Well it's a number of things. The most obvious one is when a patient hasn't improved anymore. If they're not going to improve anymore then nine times out of ten it's not appropriate for them to continue. It's time to look for somewhere else.

\section{Discussion}

Our findings suggest significant contrasts in the nature of the MDT's communication about the patient's problems and progress and with patients and their families. Where shared and commonly understood measurement tools were used (Unit 1), reporting based on the elements and scale descriptors of the measurement tools enabled a rapid description of a patient's problems to be shared and agreed. They provided a 'neutral ground' on which discrepancies in perspective could be resolved to arrive at a consensus view. This left more time for the discussion of issues that could not easily be summarized by measurement tools such as the patient's family and social situation.

The findings also indicate that the measurement tools supported clinical decision making in Unit 1 by providing a shared language and structure on which to base the team's judgements about the patient. By virtue of the team's shared tacit understanding of their meaning, the measurement tools allowed rapid exchange of information and facilitated achievement of a shared understanding of the patient. This enabled the team to agree on the nature and cause of patient's problems, provide a consistent means of monitoring patient progress over time and begin planning discharge at the start of the patient stay. Consequently, the team discussions about patients Unit 1 were more concise and decisions, particularly about discharge, were made more rapidly. In contrast, in Unit 2, no such common language or neutral ground existed. Discussion about the patient and development of a shared understanding was more drawn out and primarily comprised the elaboration of individual profession's viewpoints, rather than adoption of a shared perspective. Where differences in opinion or experience were expressed, they were rarely acknowledged and seemingly left unresolved and there was no consistent basis on which to judge whether the patient was changing over time. Consequently, team discussions about the patient were lengthy and decision making slow and inconsistent. Discharge planning rarely occurred at the start of the patient's 
JOBNAME: No Job Name PAGE: 8 SESS: 22 OUTPUT: Fri Sep 10 15:44:56 2010 SUM: 9D2D6508

/v2451/blackwell/journals/jep_v0_i0/jep_1555_oe

Impact of outcome measures on clinical practice

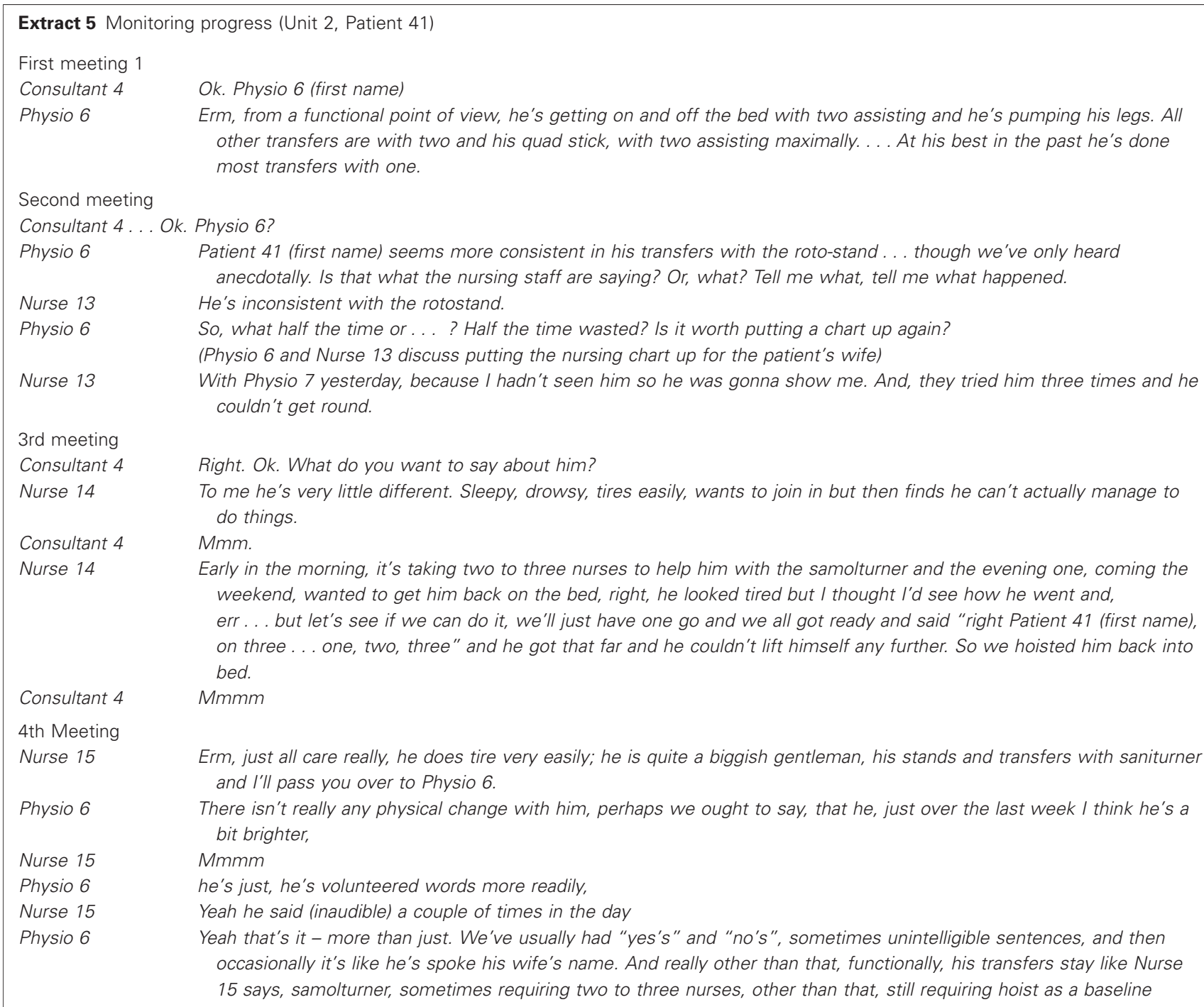

Extract 6 Predicting recovery and planning discharge (Unit 1 Patient 14)

First meeting

Consultant 2

Nurse 3

Consultant 2

Senior HO 1

Nurse 3

Consultant 1

He's high dependency. What was his Northwick Park dependency score?

Oh ok. I've got a score. It won't be high though ... in the high forties, would that seem about right?

I put his at about forty-two, something like that.

Some of his standard scores, most of his Barthel's, he's zero on every one

Yeah. The feeding, he has to be supervised because he'll choke. Erm, yeah, zero for that.

specific communication problems.
SALT Yeah that's what I got from the hand over

Consultant 2 Umm yes an early family meeting with a view to, I think unfortunately, I don't think we're going to get very far with this man.

Nurse $3 \quad$ No.

Consultant $2 \quad$ I think he's going to be destined for a nursing home.

journey but only towards the middle or end of the patient's stay in the unit.

Whether or not these contrasts impact on outcome or have implications for the quality of patient care is a subject for further research; it was not the purpose of our study to compare actual patient outcomes within the two units. Our data do suggest that using measurement tools can promote a speedier focus on the patient rather than profession-focused discussion within MDT 
meetings, thus potentially promoting patient-centred care and speeding up clinical decision making [15-17]. However, it cannot be assumed that the differences reported between the two units were solely due to the use of measurement tools; other structural, clinical and political differences could also have had an influence.

Given the observational nature of the study, care must be taken in generalizing our findings to other settings and specialties. Our observation was limited to MDT meetings and thus we can make no claims about the nature of communication and decision making outside these meetings, which has been found to have an important influence on MDT working [21]. However, one of the study's strengths is its detailed and extended observation of a set of MDT meetings. Selection of Unit 1 was purposively made as it had used measurement tools for an extended period of time. This is particularly significant given the current political drive within the UK to introduce PROMs as an indicator of quality of care [6,7] as their introduction is likely to be problematic and ultimately ineffective if made without regard to clinicians' ownership of the use of such tools, the feasibility of use and relevance to team decision making $[10,22]$. This issue has wider implications in the context of current policy directions regarding the development and adoption of quality metrics in England, arising from the Darzi review, High Care Quality for All [7].

To the authors' knowledge, this is the first study to observe the use of measurement tools in day-to-day clinical communication within MDT meetings and to compare units that do and do not use such measurement tools. Further work is needed to explore the impact of measurement tools on teamworking, quality of care, patient outcomes and on how to most effectively introduce measurement tools within practice to support an outcomes-oriented practice culture [10,22].

\section{Conclusions}

Using standardized measurement tools can promote a patientfocused (as opposed to profession-focused) approach to multidisciplinary discussions within MDT meetings, thus facilitating treatment planning and clinical decision making.

\section{Acknowledgements}

The authors acknowledge and thank the staff of the participating units for allowing us access to their meetings and for giving the time to be interviewed, and the Economics and Social Research Council (ESRC Small Grant RES-000-22-1117) for funding the study.

\section{References}

1. Rudd, A., Pearson, M. \& Georgiou, A. (2000) Measuring Clinical Outcome in Stroke (Acute Care). London, UK: Clinical Effectiveness and Evaluation Unit, Royal College of Physicians.

2. Intercollegiate Stroke Working Party (2008) National Clinical Guidelines for Stroke, $3^{\text {rd }}$ edn. London UK: Royal College of Physicians.

3. Chartered Society of Physiotherapy (2000) Core Standards of Physiotherapy Practice. London, UK: Chartered Society of Physiotherapy.

4. Department of Health (2005) The National Service Framework for Long Term Conditions. London: Department of Health. Available at: http://www.dh.gov.uk/en/Publicationsandstatistics/Publications/ PublicationsPolicyAndGuidance/DH_4105361

5. Royal College of Physicians (2006) National Sentinel Stroke Audit Report 2005. Prepared on behalf of the Intercollegiate Stroke Working Party. London, RCP.

6. Department of Health (2008) Guidance on the Routine Collection of Patient Reported Outcome Measures (Proms). London: Department of Health.

7. Department of Health (2008) High Quality Care for All. NHS Next Stages Review Final Report. (The Darzi Report). London: Department of Health. Available at: http://www.dh.gov.uk/en/ publicationsandstatistics/publications/publicationspolicyandguidance/ DH_085825 (last accessed February 2010).

8. Appleby, J. \& Devlin, N. (2004) Measuring Success in the NHS. London: King's Fund.

9. Department of Health (2008) Framing the Contribution of Allied Health Professionals: Delivering High-Quality Healthcare. London: Department of Health.

10. Greenhalgh, J. (2009) The applications of PROs in clinical practice: what are they, do they work, and why? Quality of Life Research, 18, 115-123.

11. Greenhalgh, J., Long, A. F. \& Flynn, R. (2005) The use of patient reported outcome measures in routine clinical practice: lack of impact or lack of theory? Social Science and Medicine, 60 (4), 833-843.

12. Beckerman, H., Roelofsen, E. E., Knol, D. L. \& Lankhorst, G. J. (2004) The value of the Rehabilitation Activities Profile as a quality sub-system in rehabilitation medicine. Disability \& Rehabilitation, 26 (7), 387-400.

13. Verhoef, J., Toussaint, P. J., Zwetsloot-Schonk, J. H., Breedveld, F. C., Putter, H. \& Vlieland, T. P. M. V. (2007) Effectiveness of the introduction of an International Classification of Functioning, Disability and Health-based rehabilitation tool in multidisciplinary team care in patients with rheumatoid arthritis. Arthritis \& Rheumatology, 57, 240248.

14. Wressle, E., Lindstrand, J., Neher, M., Marcusson, J. \& Henriksson, C. (2003) The Canadian Occupational Performance Measure as an outcome measure and team tool in a day treatment programme. Disability \& Rehabilitation, 25 (10), 497-506.

15. Tyson, S. F., Greenhalgh, J., Long, A. F. \& Flynn, R. (2010) The use of measurement tools in clinical practice; an observational study of neurorehabilitation. Clinical Rehabilitation, 24, 74-81.

16. Greenhalgh, J., Long, A. F., Flynn, R. \& Tyson, S. (2008) "It's hard to tell". The challenges of scoring patients on standardised outcome measures by multidisciplinary teams: a case study of Neurorehabilitation. BMC Health Services Research, 8, 217.

17. Greenhalgh, J., Flynn, R., Long, A. F. \& Tyson, S. (2008) The use of standardised outcome measures in decision making in multidisciplinary teams: a case study of Neurorehabilitation. Social Science and Medicine, 67, 183-194.

18. Skinner, A. \& Turner-Stokes, L. (2006) The use of standardized outcome measures in rehabilitation centres in the UK. Clinical Rehabilitation, 20, 609-615.

19. Charmaz, K. (2006) Constructing Grounded Theory. London: Sage.

20. Strauss, A. \& Corbin, J. (1998) Basics of Qualitative Research. London: Sage.

21. Clarke, D. J. (2010) Achieving teamwork in stroke units: The contribution of opportunistic dialogue. Journal of Interprofessional Care, 24 (3), 285-297.

22. Long, A. F. \& Jefferson, J. (1999) The significance of outcomes within European health sector reforms: towards the development of an outcomes culture. International Journal of Public Administration, 22 (3 \& 4), 385-424. 